\title{
Agents of Things (AOT): Utilizing JADE Agent Technology as Communication Middleware for Vehicle Monitoring System
}

\author{
Mohd Khidhir Eddy, Azhana Ahmad and Alicia Y. C. Tang \\ College of Computer Science and Information Technology \\ Universiti Tenaga Nasional, Malaysia \\ khidz93@gmail.com, \{azhana, aliciat\}@uniten.edu.my
}

\begin{abstract}
The Internet of Things (IoT) can be considered as a new concept that bears vast potentials as well as offering values in research studies, industrial advancements, and economical advantages. Various eminent features exist in this concept but is flawed in terms of intelligence, security, interoperability as well as availability. To solve the intelligent aspect of the concept, the existence of the concept of Agents of Things (AoT) came to fruition which utilizes software Agent technology in the concept of IoT. However, AoT is a work-in-progress concept as there are no real implementations yet. There exists technologies to implement mobile Agents. In this paper, we are looking into the communication aspects of a multi-agent system by utilizing JAVA Agent Development Framework (JADE) which will connect all the Agents for communication purposes. The system is based on the concept of Agents of Things (AoT). Agents are embedded in cars as well as the monitoring system, which continuously monitor and wait for a communication request from the cars' Agents. If an accident occurs, the Agents in the cars will communicate with the monitoring system and inform about it. This action triggers the monitoring system to contact authority and warn other vehicles heading towards the area where the accident happened. The main implementation step is installation of JADE container in devices that acts as a monitoring system. This serves as the communication hub for all Agents to connect. An application was developed and installed in portable devices (hand-phones) which act as vehicle Agents. The application has been tested and results show that the vehicle Agents can communicate with each other seamlessly and the required information (e.g. vehicle number and speed) can be transmitted successfully thus proving the concept of AoT.
\end{abstract}

Keywords: Agents of Things, Internet of Things, Self-reasoning, M2M, Mobile Agents

\section{Introduction}

The Internet of Things (IoT) can be identified to be the upcoming milestone in development of the Internet. It can be expected that this will transform the communications as well as the interactions involved between the real world and the cyber world. This can be done by linking every "Things" in the world to the Internet [1]. Devices can establish communications and connectivity with each other without any human being's intervention. This is called Machine to Machine Interaction (M2M) [2]. This enables researchers to conduct investigations in building viable systems that assists humans in various aspects in their daily life. Although the IoT can be seen as a breakthrough in advancements of the Internet, it is marred with lack of intelligence as well as self-reasoning on their environments [3]. This can be addressed with the introduction of the concept of Agents of Things (AoT) which was discussed in [4]. The

Received (August 10, 2017), Review Result (November 15, 2017), Accepted (November 24, 2017) 
concept of AoT can be predicted to be a progress in the research field towards new heights by augmenting machine to machine interactions.

Previous works on AoT development can be seen in [5] where it discusses on the computing hardware analysis for AoT as well as [6] where it discusses on the software analysis for AoT application. This paper presents the work-in-progress of the research in Agents of Things and the initial works as well as experimentation of the application developed to prove that mobile Agents technologies can ease communications between authorities and users to hasten the process of rectifying accidents or breach of laws. We organize the paper as follows: Section II reviews similar work. Section III reviews technologies use for mobile Agent communications. Section IV presents the implementation scenario of the mobile Agents and Section V concludes the paper.

\section{Related Work}

Mobile Agents are programs that travel through a means of computer communications networking and are able to persistently identify itself and its local state. Agents can communicate with one another and other mobile Agents or with a stationary Agent. Furthermore, these Agents are able to co-exist with information or processing resources, propagating data as well as conducting businesses on behalf of their respected users [7]. The next few sections highlight the Agent background and its current reality and challenges.

\subsection{Agent Background}

A particular Agent acts as an entity that functions to run routine tasks ordered by its users and to achieve a planned goal within the context of a set environment. The main modification between an Agent and a traditional software entity is that traditional software functions to its designed procedures as well as macros to run determined codes. While Agents integrates the capability to administer intelligence by carrying out autonomous/semi-autonomous decisions in relation to its dynamic runtime situations [8]. The central representation of an Agent is called Autonomy. Commonly, an Agent is an encapsulated system which is placed in certain specific environment and is capable of flexible, autonomous act in the environment to meet its intended purposes [8]. There exists numerous application domains of software system functions that are suitable for Agent techniques such as below in Table 1.

Table 1. Agent Applications Catalogs [8]

\begin{tabular}{|l|l|}
\hline Application Domain & Application Fields \\
\hline Industrial & $\begin{array}{l}\text { Air Traffic Control, Network and Telecommunication Management, } \\
\text { Transportation System, Critical Process Control, Critical Process Control }\end{array}$ \\
\hline Commercial & Information Management, Business Process Management. Electronic Commerce \\
\hline Medical & Health Care, Patient Monitoring \\
\hline Entertainment & Interactive Theater and Cinema, Video Games \\
\hline
\end{tabular}

One of the recent applications of multi-agent using IoT can be found in [9] where it discussed an Agent-based middleware architecture for reconfigurable manufacturing systems. Recent Object-Oriented software engineering procedures offer tools and method for developing a traditional software systems. Modeling, analyzing, designing and testing acts as a standard for Object-Oriented software. Development of Agent systems is a nontrivial task without the necessary infrastructure. Developing Agent-Oriented software necessitates methodologies and tools to integrate steps from problem realization, architecture design, requirement analysis as well as implementations. 


\section{JADE Agent Technology and Its Significance in the Vehicle Monitoring System}

As was mentioned, the technology used to implement the vehicle monitoring's communications. It acts as a middleware for the development as well as the run-time execution of peer-to-peer application which was developed and are based on the agents' paradigm and can seamlessly work and interoperate both in wired and wireless environment [10]. Such application can be seen in [11] where it displays a distributed system and multi-agent system (MAS) that enables effective and efficient management of complex distribution network. JADE was used as it fits the simulation of such system and enables simulation of the physical and communication models concurrently.

The compatibility of JADE with various platforms and field testing with utilization of old mobile phone models has been discussed in [12]. JADE is extremely versatile and fits the constraints of environments with limited resources which is crucial. Furthermore, complex architectures such as .NET or JAVA 2 Platform Enterprise Edition (J2EE) has been integrated [13]. In accordance to this, JADE acts as a service to perform multi-party proactive programs as well as applications. JAVA-enabled mobile phones are easily installable with JADE due to the limited memory footprint. This includes current generation mobile phones with better technology capabilities. Figure 1 shows the basis of JADE architecture which illustrates the layers involved.

JADE provides necessary services to distribute peer-to-peer applications in the fixed environment as well as the mobile agents installed in the vehicles. JADE also allows each of the agents to vigorously discover other agents and communicate with them accordingly. Agents communicate by exchanging of asynchronous messages which is easily transmittable to all devices for dispersed and loosely coupled communications. For the communication to take place, an agent transmits a message to a destination which they are identified by a name and there exists no temporal reliance between communicating agents. Further explanation can be seen in [10].

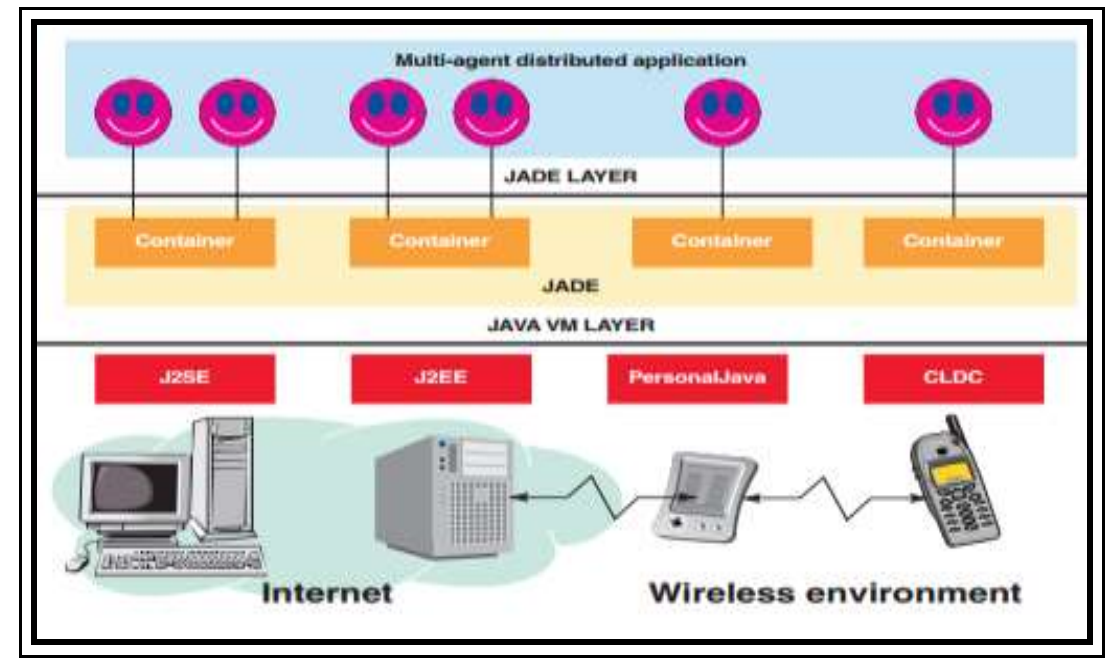

Figure 1. The JADE Architecture [10]

\subsection{JADE in Mobile Environment}

LEAP is a module which enables optimizing of all communication mechanisms when interacting with devices with limited resources and are connected through wireless networks. Activating this module renders a JADE container to "split", as depicted in Figure 2 below into a front-end running on the mobile terminal, and a back-end running in the fixed network [10]. 


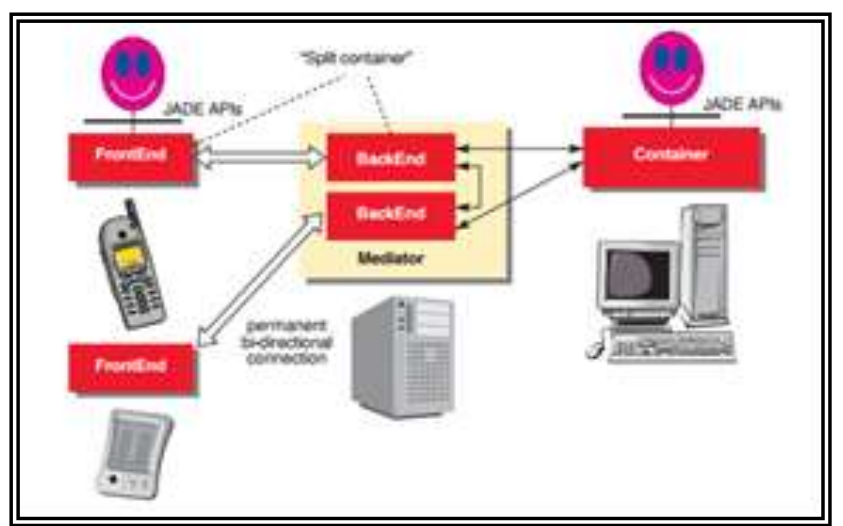

Figure 2. JADE Architecture in the Wireless Environment [10]

Mediator, an architectural element essentially needs to be active. It is responsible for instantiating and holding the back-ends. It can also be deployed more than a single mediator holding several back-ends to handle work-load issues. Each front-end is linked to its corresponding back-end by means of a permanent bi-directional connection [10]. It is imperative to take note of that there is no distinction at all for application engineers relying upon whether an agent is conveyed on a typical container or the front-end of a split container, since both the accessible usefulness and the APIs to get to them are precisely the same. The approach has various points of interest which are valuable in actualizing the vehicle monitoring system:

- The front-end of the container is extremely lightweight in terms of required memory and processing power. Furthermore, The front-end is able to detect a loss of connection with the back-end and re-establish it as soon as possible

- A few data that containers trade (for example to recover the container where an agent is at present running) are taken care of just by the back-end. This approach, together with somewhat proficient encoding of interchanges between the front-end and the back-end, permits upgrading the utilization of the remote connection.

\subsection{Implementation of JADE as Middleware for the Vehicle Monitoring System}

To visualize the Vehicle Monitoring System (VSM), an initial work or testing of communication between devices or Things has been done. The platform used is Android and the tested hardware are a smartphone and a laptop. The framework or technology used to develop and handle software agents is called Java Agent Development Framework (JADE). JADE is a software framework that is fully implemented using the Java language. It simplifies implementation of multi-agent systems by utilizing a middle-ware which complies with FIPA specifications with varied graphical tools to support debugging as well as deployment [14]. Both Things are embedded with a software agent called ChatAgent which handles chat or messages with agents that are connected to a single container. The agents will be recognizing each other's Internet Protocol (IP) addresses in the container therefore establishing connection and can communicate with each other. Demonstrations can be seen as below. 


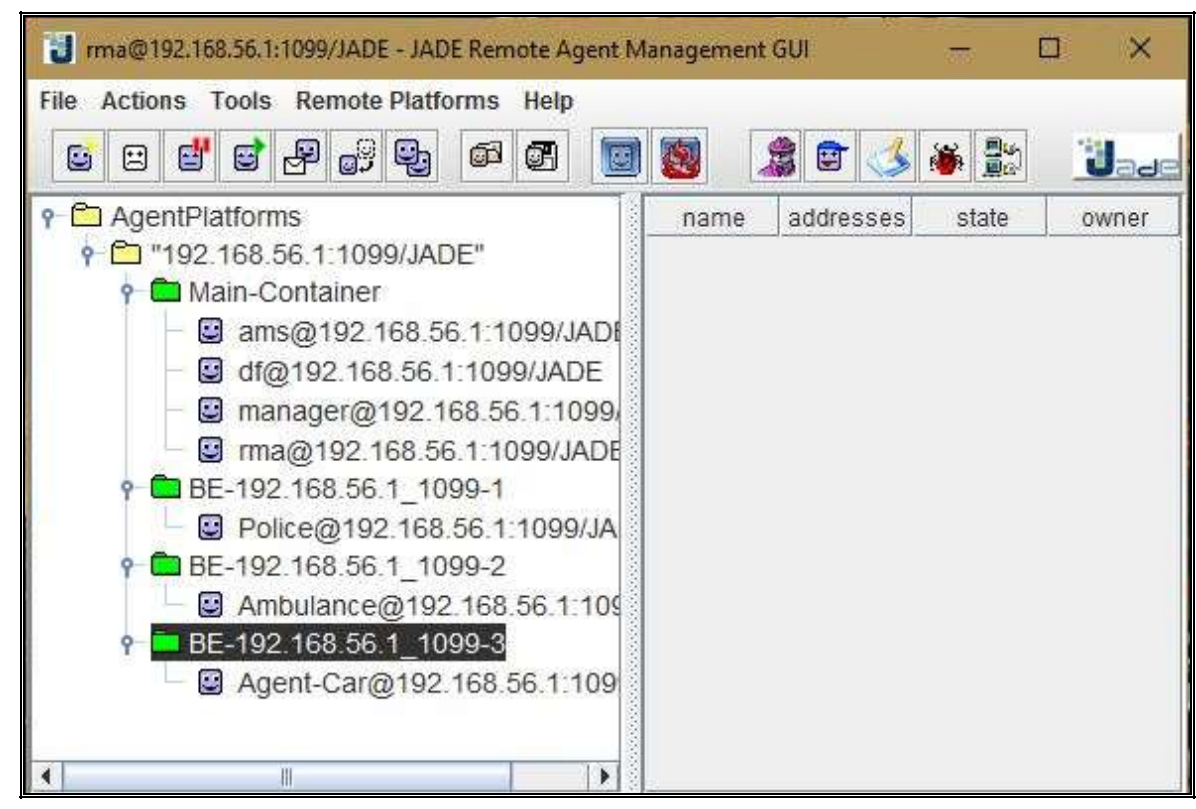

Figure 3. JADE Agent Container

Figure 3 shows the JADE Agent container which acts as hub of communication between agents from varied devices. For this experimentation, the devices involved is a smartphone and a laptop which is connected to the same wireless network. The container was started at the laptop (which acts as the communication hub between other mobile agents) hence the IP shown above (192.168.56.1). Figure 4 below shows interface of the service agents such as Police and Ambulance which they will receive messages instantaneously and messages they sent will be transmitted to all agents for immediate response. The container also shows all connected agents. The "Main -Container" contains services to run the JADE container. The notable ones are Agent Management System (AMS) controls the platform and has the ability to create and destroy connected agents, containers and stopping the platform. Directory Facilitator (DF) which provides directory to announce which agents are available on the platform. Lastly the Remote Management Agent (RMA) which is the agent implementing the JADE Management Console itself [14].

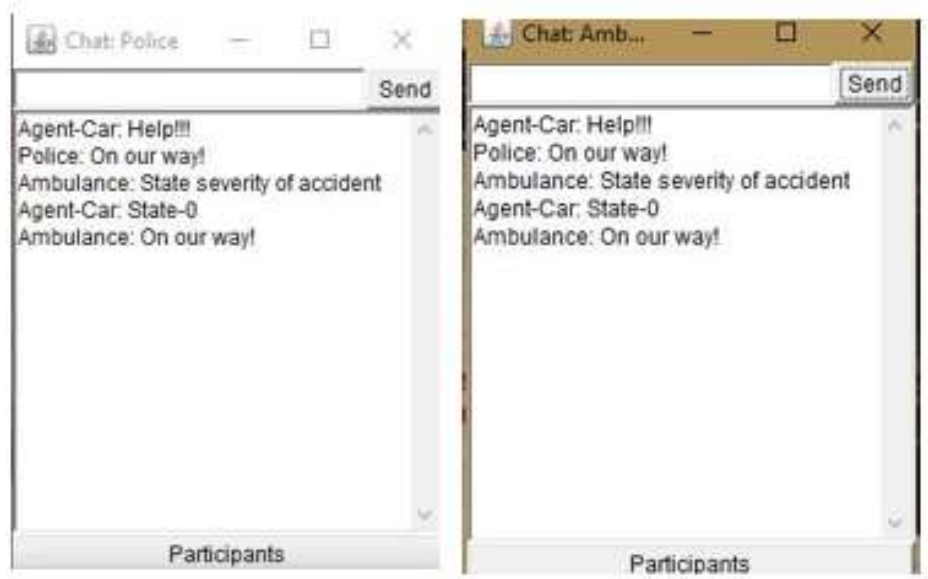

Figure 4. Participating Agent in the Network with their Respective Interfaces

Figure 5 shows the interface of the application developed on the smartphone (mobile agents) for vehicle agents after it is connected to the container of the communication hub. As seen in the figure, the interface shows all messages are the same on all the agents 
which indicates seamless information transmission which is crucial in handling accidents as well as law-abiding issues. Mobile Phones used were a Asus Zenfone 2 as well as a Samsung J5 which indicates JADE can be used on older or new mobile phones with JAVA capabilities.

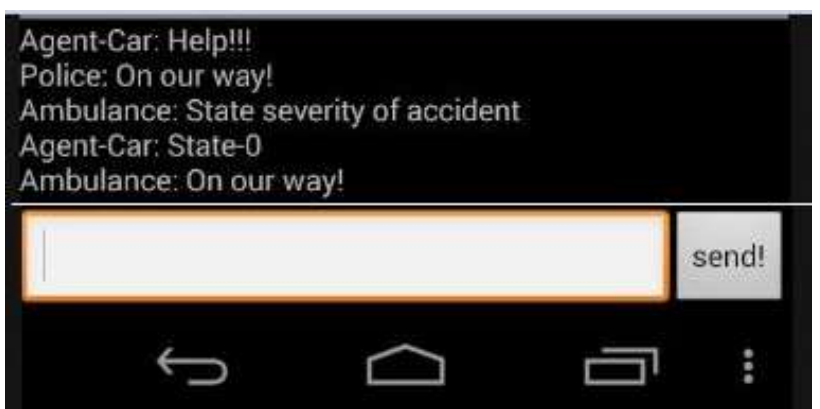

Figure 5. Agent from Mobile Phone Interface

\section{Application Scenario of JADE Agent Technology and Mobile Application}

Executing the AoT idea, all things considered, can yield many advantages, rectifying many issues and eases our daily routines. With JADE acting as the middleware for this system, the communication aspect of the system has been looked into. In the previous section, the purpose of such example is to be used in the following scenario. We exhibit a run of the mill situation of a street movement as an example to show transmission of messages to ease the lives of people and parties involved in road accidents. The explanation of such scenario can be seen in [4]. We will focus on the transmission of messages in the scenario.

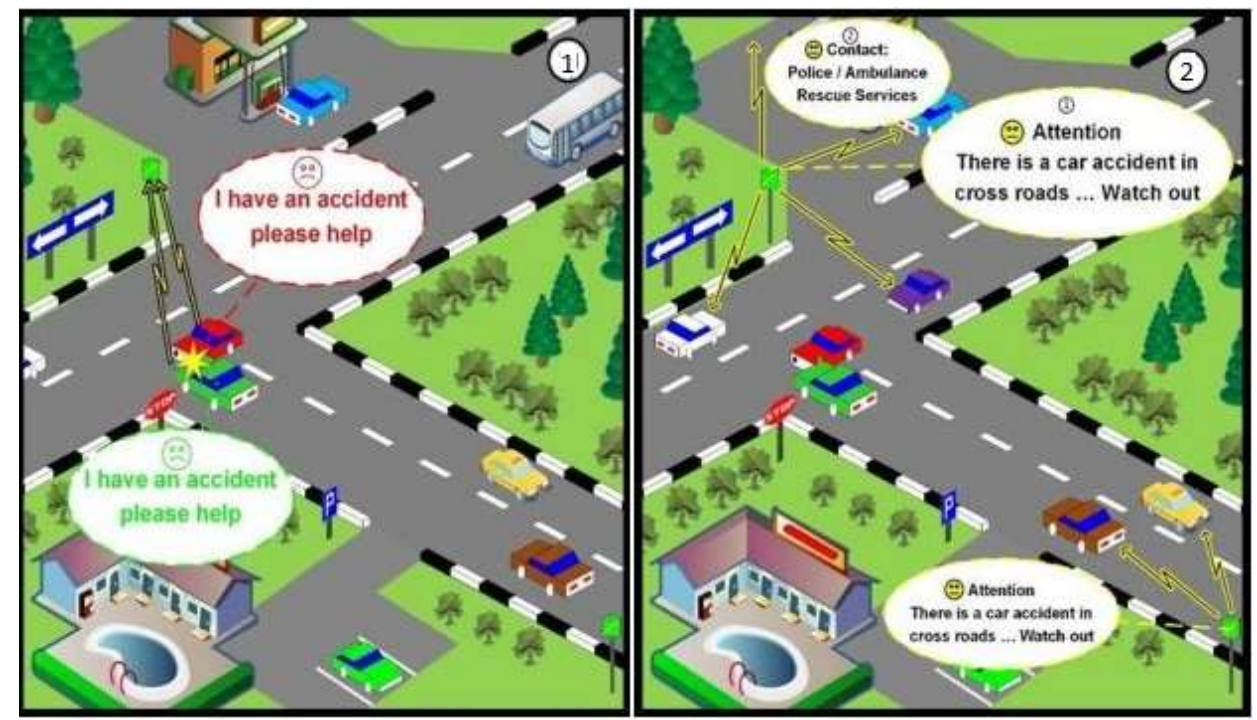

Figure 6. The Road Accident Scenario (1) and (2) [4]

In Figure 6, the arrows pointing towards an object is the monitoring system which acts as a communications hub as mentioned in the previous section. When a road accident happens, the vehicles that are engaged with the mishap will illuminate the monitoring system through their agents with a message as above. The monitoring system will continuously warn other vehicles approaching the scene of accident. As mentioned in previous section, the monitoring system will inform all the agents on the accident and 
messages will transmit instantaneously among all the connected agents (Police, Ambulance, Drivers, etc.). The advantages of caution drivers about mishaps and constantly observing their speed rates out and about help to spare lives. Sequentially, the above mentioned scenario also shows the monitoring system informs the service agents for immediate response on the situation.

\section{Conclusion and Further Work}

In this paper, we review the Agent technology background and related work regarding mobile Agent as well. Furthermore, we have also reviewed JADE Agent technology and proved its significance as the middleware to the communications aspect of Agents of Things and the aforementioned scenario. We also proposed the JADE to be used in JAVA-powered mobile phones to act as Agents in the multi-agent system and demonstrate the applicability of the concept in easing the communication issues associated with AoT's concept. In our future work, we shall proceed to enhancing the user interface of the application developed with the JADE technology to make it more userfriendly as well as identifying the hardware to be used in creating a portable device which can be embedded in a vehicle with the application developed to be installed in it.

\section{Acknowledgments}

The authors of this paper would like to express our upmost gratitude to the Ministry of Education for supporting and funding this research under the Fundamental Research Grant Scheme (FRGS). Furthermore, we extend our appreciation to the fellow lecturers and researchers from the College of Computer Science and Information Technology, Universiti Tenaga Nasional.

\section{References}

[1] A. Zaslavsky and D. Georgakopoulos, "Internet of Things: Challenges and State-of-the-Art Solutions in Internet-Scale Sensor Information Management and Mobile Analytics", In Mobile Data Management (MDM), 2015 16th IEEE International Conference on IEEE, (2015), pp. 3-6.

[2] J. Jermyn, R.P. Jover, I. Murynets, M. Istomim and S. Stolfo, "Scalability of Machine to Machine Systems and the Internet of Things on LTE mobile networks", In World of Wireless, Mobile and Multimedia Networks (WoWMoM), 2015 IEEE 16th International Symposium on a IEEE, (2015), pp. $1-9$.

[3] L. Tan and N. Wang, "Future internet: The internet of things", Advanced Computer Theory and Engineering (ICACTE), 2010 3rd International Conference on, vol. 5, (2010), pp. V5-376-V375-380.

[4] A.M. Mzahm, M.S. Ahmad and A.Y. Tang, "Agents of Things (AoT): An intelligent operational concept of the Internet of Things (IoT)", In Proceedings of the Intelligent Systems Design and Applications (ISDA), 2013 13th International Conference on (2013), IEEE, (2013), pp. 159-164.

[5] A.M. Mzahm, M.S. Ahmad and A.Y. Tang, "Computing hardware analysis for Agents of Things (AoT) applications", In Proceedings of the Information Technology and Multimedia (ICIMU), 2014 International Conference on (2014), IEEE, (2014), 223-228.

[6] A.M. Mzahm, M.S. Ahmad, A.Y. Tang and A. Ahmad, "Software Analysis for Agents of Things (AoT) Applications", International Symposium on Agents, MultiAgent Systems and Robotics (ISAMSR 2015), (2015).

[7] M. Friedemann and K. Rothernel, "Mobile Software Agents", Workshop at Schloss Dagstuhl, (1997).

[8] L. Chie-En and M.K. Krishna, "A Methodology to Evaluate Agent Oriented Software Engineering Techniques", The International Journal of Multiagent and Grid Systems (20052005).

[9] R. Priego, N. Iriondo, U. Gangoiti and M. Marcos, "Agent-based middleware architecture for reconfigurable manufacturing systems", International Journal of Advanced Manufacturing Technology, vol. 92, (2017), pp. 1579-1590.

[10] F. Beliferniine, G. Caire and A. Poggi, “JADE - A White Paper”, Exp., vol. 3, no.3, (2003).

[11] Y. Duan, L. Luo, T. Li, Y. Cao, C. Rehtanz and M. Kuch, "Co-simulation of distributed control system based on JADE for smart distribution networks with distributed generations", IET Generation, Transmission \& Distribution, (2017), pp. 1751-8695.

[12] M. Berger, S. Rusitschka and D. Toropov, "Porting Agents to Small Mobile Devices", The Development of the Lightweight Extensible Agent Platform.

[13] BlueJADE. Retrieved from http://sourceforge.net/projects/bluejade. 
[14] G. Caire, "Jade Tutorial: Jade Programming for Beginners", Retrieved August 24, 2016, from jade.tilab.com: http://jade.tilab.com/doc/tutorials/JADEProgramming-Tutorial-for-beginners.pdf(2009, June 30).

\section{Authors}

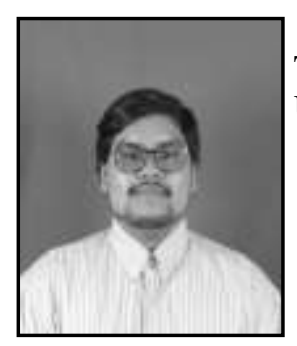

Mohd Khidhir bin Eddy Yusrizal, he is a student in Master Information Technology, College of Computer Science and Information Technology, Universiti Tenaga Nasional, Malaysia.

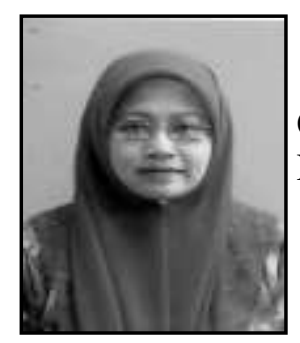

Azhana Ahmad (PhD), she is a senior Lecturer, College of Computer Science and Information Technology, Universiti Tenaga Nasional, Malaysia.

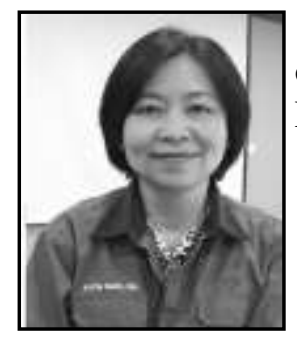

Alicia Tang Y. C. (PhD), she is an Associate Professor, College of Computer Science and Information Technology, Universiti Tenaga Nasional, Malaysia. 